International Journal of Pure and Applied Mathematics

Volume 117 No. 1 2017, 81-86

ISSN: 1311-8080 (printed version); ISSN: 1314-3395 (on-line version)

url: http://www.ijpam.eu

doi: 10.12732 /ijpam.v117i1.8

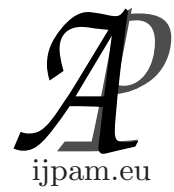

\title{
JULIA SETS AS COMPLEX POLYNOMIAL
}

\author{
M. Senthamaraikannan ${ }^{1 \S}$, R. Kamali ${ }^{2}$, G. Jayalalitha ${ }^{3}$ \\ ${ }^{1,2,3}$ Department of Mathematics \\ Vels University \\ Chennai, 600 117, Tamil Nadu, INDIA
}

\begin{abstract}
Julia sets are certain fractal sets in the complex plane that arise from the dynamics of complex polynomials and it characterize by the structure (i.e., boundary) is the main aim to discuss the complex polynomial.
\end{abstract}

AMS Subject Classification: 37F10, 37F50, 30D45, 37C25

Key Words: polynomials, Julia set, normal family, periodic points

\section{Introduction}

Complex dynamics [1] uses techniques from many fields, especially complex analysis. The Julia set of a rational map is the chaotic part of the dynamics. The structure of Julia sets, which are the sets in which a complex function behaves chaotically, and which are often fractals. It is a field whose first explorers included Gaston Julia and Pierre Fatou at the turn of the twentieth century, but which made its largest gains in the 1980s with the use of computers to discover the beauty of Julia sets and the Mandelbrot set.

First, we try to determine if the Julia set [2] takes up any space, in the sense of having a Lebesgue measure greater than zero. If $f: \mathbb{C} \rightarrow \mathbb{C}$ is a polynomial function, the filled Julia set of $f$ is the set of points whose orbits are bounded. Julia set of $f$ denoted by $J_{f}$ is the boundary of the Julia set. A set which looks like a curve on the screen but takes up space would be extremely complex (Fig. 1(a))[3]. However, since most known Julia sets do not take up space, we need to be able to classify the complexity by different means.

$\begin{array}{lr}\text { Received: } & 2017-06-08 \\ \text { Revised: } & 2017-07-13 \\ \text { Published: } & \text { November } 28,2017 \\ \text { Correspondence author }\end{array}$

Received:

Revised:

$\S_{\text {Correspondence author }}$
(C) 2017 Academic Publications, Ltd. url: www.acadpubl.eu 
The characterisations of what we might term a Julia set. The first being that the Julia set $J(f)$ of a function $f$ is the set of all points $z$ in $\mathbb{C}$ such that $f$ displays sensitive dependence at $z[4]$. In other words, $J(f)$ is the sets of all Chaotic points of $f$. This definition could be used in the study of arbitrary functions $f: \mathbb{C} \rightarrow \mathbb{C}$ but for the purposes of this report we will only be concerned with functions which are holomorphic i.e functions which are complex-differentiable for all $z \in \mathbb{C}$.

Devaney's [5] characterization of a Julia set is based on classifying points in the complex plane according to their long term behaviour under repeated application of $f$. A distinction is made between points $z$ for which $f^{n}(z)$ remains bounded as $n \rightarrow \infty$ and points for which $f^{n}(z)$ diverges. The Julia set is then defined to be the boundary between these two types of behaviour (Fig. 1(b)).

In this paper, in Section 2 preliminaries and definitions are given. In Section 3 the characterization of Julia set based on normal family and repelling.

\section{Preliminaries}

Definition 2.1 (Connectedness). Let $S$ be a subset of the complex plane.

- Two points $p, q \in S$ are connected by a path in $S$ if there exists a continuous path lying entirely in $S$ that begins at $p$ and ends at $q$.

- If every pair of points in $S$ are connected by a path in $S, S$ is pathconnected. Otherwise, $S$ is disconnected.

Definition 2.2 (Path component). Let $S$ be a subset of the complex plane, and let $p \in S$. The path component of $S$ containing $p$ is the set

$$
\{q \in S \mid p \text { and } q \text { are connected by a path in } S\}
$$

That is, the path component of $S$ containing $p$ is the "piece" of $S$ that contains $p$.

Definition 2.3 (Topological space). If $S$ is a subset of topological space $X$ then a neighbourhood of $S$ is a set $V$ that includes an open set. $U$ containing $S$. it follows that a set $V$ is a neighbourhood of $S$ if and only if it is a neighbourhood of all the points in $S$.

Definition 2.4 (Normal family). The family $\left\{g_{k}\right\}$ is normal at the point $w$ of $U$ if there is some open subset $V$ of $U$ containing $w$ such that $\left\{g_{k}\right\}$ is a normal family on $V$. 
Definition 2.5. The Julia set of the function $f$ is defined to be the boundary of

$$
J(f)=\partial K(f)
$$

Definition 2.6. The filled in Julia set of the function $f$ is defined as

$$
K(f)=\left\{z \in \mathbb{C}: f^{k}(z) \rightarrow \infty\right\}
$$

Definition 2.7 (Repelling). The study of sequences $f^{k}(z)$ for various initial $z$ is known as complex dynamics. the position of $z$ relative to the Julia set $J(f)$ is a key to this behaviour.

Definition 2.8 (Perfect set). A set with closed and with no isolated points is called as perfect set.

Theorem 2.9 (Montel's theorem). Let $\left\{g_{k}\right\}$ be a family of complex analytic functions on an open domain $U$. If $\left\{g_{k}\right\}$ is not a normal family then for all $w \in \mathbb{C}$ with at most one exceptions we have $g_{k}(z)=w$ for some $z \in U$ and some $k$.

\section{The Characterization of Julia Set}

Theorem 3.1. For a polynomial $f$, the Julia set $J(f)$ is the closure of the repelling periodic points of $f$.

Proof. Let $w$ be a repelling periodic point of $f$ of period $p$, So $w$ is a repelling fixed point of $g=f^{p}$. Suppose that $\left\{g^{k}\right\}$ is normal at $w$, then $w$ has an open neighbourhood $V$ on which a subsequence $\left\{g^{k_{i}}\right\}$ converges to a finite analytic function $g_{0}$ (it cannot converge to $\infty$ since $g^{k}(w)=w$ for all $k$ ). By a standard result from complex analysis, the derivatives also converge, $\left(g^{k_{i}}\right)^{\prime}(z) \rightarrow g_{0}^{\prime}(z)$, for $z \in V$. However, by the chain rule, $\left|\left(g^{k_{i}}\right)^{\prime}(w)\right|=\left|\left(g^{\prime}(w)\right)^{k_{i}}\right| \rightarrow \infty$. Since $w$ is a repelling fixed point and $\left|g^{\prime}(w)\right|>1$. This contradicts the finiteness of $g_{0}^{\prime}(w)$, so $\left\{g^{k}\right\}$ cannot be normal at $w$. Thus, $w \in J(g)=J\left(f^{p}\right)=J(f)$ using " $J\left(f^{P}\right)=J(f)$ for every positive integer $P$." Since $J(f)$ is closed, it follows that the closure of the repelling periodic points is a subset of $J(f)$.

Let $E=\{w \in J(f)$ such that there exists $v \neq w$ with $f(v)=w$ and $\left.f^{\prime}(v) \neq 0\right\}$ suppose that $w \in E$. Then, there is a open neighbourhood $V$ of $w$ on which we may find a local analytic inverse $f^{-1}: V \rightarrow \mathbb{C} \backslash V$, so that $f^{-1}(w)=v \neq w$ (just choose values of $f^{-1}(z)$ is a continuous manner). Define a family of analytic function $\left\{h_{k}\right\}$ on $V$ by

$$
h_{k}(z)=\frac{\left(f^{k}(z)-z\right)}{\left(f^{-1}(z)-z\right)} ;
$$


provided $V$ is chosen to be a sufficiently small neighbourhood of $w$ the denominator will be non-zero.

Let $U$ be any open neighbourhood of $w$ with $U \subset V$. Since $w \in J(f)$, the family $\left\{f^{k}\right\}$ and thus, from the definition, the family $\left\{h_{k}\right\}$ is not normal on $U$. By Montel's Theorem :2.9 $h_{k}(z)$ must take either the value 0 or 1 for some $k$ and $z \in U$. In the first case, $f^{k}(z)=z$ for some $z \in U$; in the second case, $f^{k}(z)=f^{-1}(z)$, so $f^{k+1}(z)=z$ for some $z \in U$. Thus, $U$ contains a periodic point of $f$, so $w$ is in the closure of the repelling periodic points of $f$ for all $w \in E$. Since $f$ is a polynomial, $E$ contains all of $J(f)$ except for a finite number of points. Since $J(g)$ contains no isolate points, by " $J(f)$ is a perfect set (i.e. closed and with no isolated points) and is therefore uncountable

(i) $V$ is not a fixed or periodic point of $f$. By corollary "If $z \in J(f)$, then $J(f)$ is the closure of $\bigcup_{k=1}^{\infty} f^{-k}(z)$ " and proposition, "The Julia set $J=J(f)$ of $f$ is forward and backward invariant under $f$, that is, $J=f(J)=f^{-1}(J)$ ", $U$ contains a point of $f^{-k}(V) \subset J(f)$ for some $k \geq 1$, and this point must be different from $V$.

(ii) $f(V)=V$. If $f(z)=V$ has no solution other than $V$, then, just as in the proof of "Let $f$ be a polynomial, let $w \in J(f)$, and let $U$ be any neighbourhood of $w$. Then, for each $j=1,2, \ldots$, the set $W \equiv \bigcup_{k=j}^{\infty} f^{k}(U)$ is the whole of $\mathbb{C}$, except possibly for a single point. Any such exceptional point is not in $J(f)$, and is independent of $w$ and $U$ ", $V \notin J(f)$. Thus, there exists $w \neq v$ with $f(w)=v$. By

a) The following holds for all $z \in \mathbb{C}$ with at most one exception:

If $U$ is an open set that intersects $J(f)$ then $f^{-k}(z)$ intersects $U$ for infinitely many values of $K$.

b) If $z \in J(f)$, then $J(f)$ is the closure of $\bigcup_{k=1}^{\infty} f^{-k}(z)$.

$U$ contains a point $u$ of $f^{-k}(w)=f^{-k-1}(v)$ for some $k \geq 1$. Any such $u$ is in $J(f)$ by backward invariance and is distinct from $V$, since $f^{k}(V)=$ $V \neq w=f^{k}(u)$.

(iii) $f^{P}(v)=v$ for some $P>1$. By " $J\left(f^{P}\right)=J(f)$ for every positive integer $P^{\prime \prime}, J(f)=J\left(f^{P}\right)$, so by applying (ii) to $f^{P}$, we see that $U$ contains points 


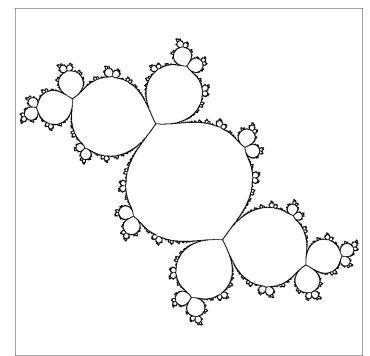

(a)

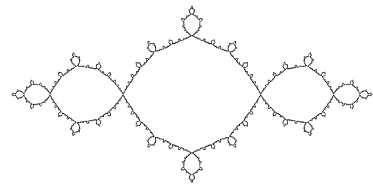

(b)

Figure 1: (a), (b) Julia set

of $J\left(f^{P}\right)=J(f)$ other than $V$. Thus, $J(f)$ has no isolated points, since it is closed, it is perfect. Finally, every perfect set is uncountable; we can now prove the main result of this section, that the Julia set $J(f)$ is the closure of the repelling periodic points of $f . "$

$J(f) \subset \bar{E}$ is a subset of the closure of the repelling periodic points.

This Montel's theorem :2.9 leads to characterizing the Julia set

Theorem 3.2. $J(f)=\left\{z \in \mathbb{C}\right.$ : the family $\left\{f^{k}\right\}$ is not normal at $\left.z\right\}$.

Proof. If $z \in J$, then in every neighbourhood $v$ of $z$, there are points $w$ such that $f^{k}(w) \rightarrow \infty$, whilst $f^{k}(z)$ remains bounded. Thus, no subsequence of $\left\{f^{k}\right\}$ is uniformly convergent on $v$, so that $\left\{f^{k}\right\}$ is not normal at $z$.

Suppose that $z \notin J$. Either $z \in$ int $k$, in which case, taking an open set $v$ with $z \in V \subset$ int $k$, we have $f^{k}(w) \in k$ for all $w \in V$ and all $k$, so by Montel's theorem $\left\{f^{k}\right\}$ is normal at $w$ otherwise, $z \in \mathbb{C} \backslash k$ so $\left|f^{k}(z)\right|>r$ for some $k$, where $r$ is given by "Given polynomial $f(z)=a_{n} z^{n}+a_{n-1} z^{n-1}+\cdots+a_{0}$ with $n \geq 2$ and $a_{n} \neq 0$, there exists a number $r$ suchthat if $|z| \geq r$, then $|f(z)| \geq 2|z|$. In particular, if $\left|f^{m}(z)\right| \geq r$ for some $m \geq 0$, then $f^{k}(z) \rightarrow \infty$ as $k \rightarrow \infty$. Thus, either $f^{k}(z) \rightarrow \infty$ or the set $\left\{f^{k}(z): k=0,1,2, \ldots\right\}$ is bounded", $f^{k}(w) \rightarrow \infty$ uniformly on $V$, so again $\left\{f^{k}\right\}$ is normal at $w$.

The expression " $J(f)=\left\{z \in \mathbb{C}\right.$ : the family $\left\{f^{k}\right\}$ is not normal at $\left.z\right\} "$ is normally taken as the definition of the Julia set in more advanced work, since it is more widely applicable and tends itself to techniques from complex analysis. Then, the theory developed from Montel's theorem extends to a wide class of complex functions including rational functions and meromorphic function. 


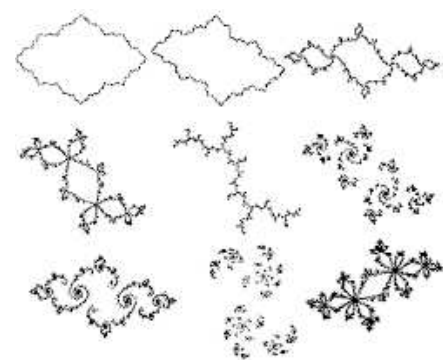

(a)

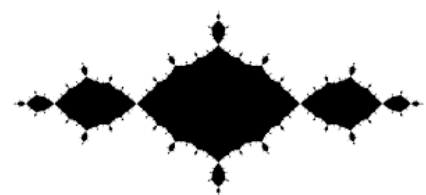

(b)

Figure 2: (a), (b) Characterization of Julia set

Note, however, that if $f: \mathbb{C} \cup(\infty) \rightarrow \mathbb{C} \cup\{\infty\}$ is a rational function, then $J$ must be closed, but need not be bounded. Indeed, it is possible for $J$ to be the whole complex plane [6].

The main aim now is to obtain a characterisation of the Julia set as the closer of the repelling a periodic points of $J(f)$. Based on Montel's theorem the Julia are characterised by Normal family and repelling (Fig. 2(a) and 2(b)). In Julia sets $f(z)=z^{2}+c$ where $c$ is complex number, this complex number gives polynomials. Hence Julia sets as complex polynomial.

\section{References}

[1] Paul Blanchard, Complex analytic dynamics on the Riemann sphere, Bulletin (New Series) of the American Mathematical Society, 11, No. 1 (1984).

[2] Gaston Julia, Memoire Sur I'iteration des functions rationnelles, Journal de mathematiques pures et appliquecs $8^{e}$ serie, 1 (1918), 47-246.

[3] H.-O. Peitgen, P.H. Richter, The Beauty of Fractals. Images of Complex Dynamical System, Springer, Berlin (1986).

[4] H.-O. Peitgen, H. Jurgens and D. Saupe, Chaos and Fractals, New Frontiers of Science, Springer-Verlag, New York Inc. (1992), doi: 101007/b97624.

[5] Devaney L. Robert, Complex dynamics of quadratic polynomial's, Complex Dynamical Systems: The Mathematics Behind the Mandelbrot and Julia Sets, 49 (1994), 1-30.

[6] Kenneth J. Falconer, Fractal Geometry Mathematical Foundations and Applications, Third Edition, Wiley, 2014. 